\title{
Specialty-care access for community health clinic patients: processes and barriers
}

This article was published in the following Dove Press journal: Journal of Multidisciplinary Healthcare

\author{
Mabel C Ezeonwu \\ School of Nursing and Health Studies, \\ University of Washington Bothell, \\ Bothell, WA, USA
}

Introduction: Community health clinics/centers (CHCs) comprise the US's core health-safety net and provide primary care to anyone who walks through their doors. However, access to specialty care for $\mathrm{CHC}$ patients is a big challenge.

Materials and methods: In this descriptive qualitative study, semistructured interviews of 37 referral coordinators of CHCs were used to describe their perspectives on processes and barriers to patients' access to specialty care. Analysis of data was done using content analysis. Results: The process of coordinating care referrals for $\mathrm{CHC}$ patients is complex and begins with a provider's order for consultation and ends when the referring provider receives the specialist's note. Poverty, specialist and referral coordinator shortages, lack of insurance, insurance acceptability by providers, transport and clinic-location factors, lack of clinic-hospital affiliations, and poor communication between primary and specialty providers constitute critical barriers to specialty-care access for patients.

Conclusion: Understanding the complexities of specialty-care coordination processes and access helps determine the need for comprehensive and uninterrupted access to quality health care for vulnerable populations. Guaranteed access to primary care at CHCs has not translated into improved access to specialty care. It is critical that effective policies be pursued to address the barriers and minimize interruptions in care, and to ensure continuity of care for all patients needing specialty care.

Keywords: community health clinics and centers, access to healthcare, barriers to health care access, specialty-care referral process, care coordination, safety-net, undeserved populations, health care system, vulnerable populations, affordable comprehensive health insurance

\section{Introduction}

In this cross-sectional descriptive study, perspectives of managerial and frontline personnel in community health clinics/centers (CHCs) in Washington State were explored regarding specialty-care referral and coordination and the challenges of access for $\mathrm{CHC}$ patients. This study provides answers to the key research questions: What is the process of referral to specialty-care (SC) services for patients seen at CHCs?; and What are the barriers of access to SC for CHC patients?

CHCs comprise the nation's core health care safety net and provide primary care (PC) to anyone who walks through their doors. Individuals served by the clinics on average have poor health status and higher prevalence of chronic disease conditions than the general population. ${ }^{1,2}$ Twenty-five percent of patients who present to these centers require specialty and diagnostic services that are not provided by the centers. ${ }^{3}$ The CHCs are limited in their abilities to provide specialty services and other important
School of Nursing and Health Studies, University of Washington Bothell, 18115 Campus Way Northeast - Box 358532,

Bothell, WA $9801 \mathrm{I}$, USA

Tel +l 4253523815

Fax +l 4253523237

Email mezeo@uw.edu 
aspects of care that go beyond PC., ${ }^{4,5}$ Evidence shows that CHC patients have reduced access to specialists, ${ }^{3,6,7}$ despite the fact that these services are critical for continued and consistent recovery or health maintenance. Regardless of patient diagnosis or socioeconomic status, care coordination and timely referrals to appropriate providers are essential. This study examines the process of SC coordination and referral and barriers that limit $\mathrm{CHC}$ patients from accessing specialty services outside the CHCs.

CHCs constitute a critical part of Washington State's health care system. In 2008, 10.1\% of the state's residents were served by CHCs. ${ }^{7}$ In 2014 and 2016, CHCs were also medical homes to 885,210 and 1,035,629 patients, respectively. ${ }^{89}$ The majority of $\mathrm{CHC}$ patients are from vulnerable populations. For example, in 2014, $21 \%(189,608)$ were uninsured, $57 \%(499,624)$ were on Medicaid, and 35\% $(308,265)$ were under 19 years old. ${ }^{8}$ Furthermore, nearly a half (49\%) of patients were visible minorities and $68 \%$ lived at or below the federal poverty level. ${ }^{7,8}$ In $2016,16 \%(165,357)$ were uninsured, $60.7 \%(628,450)$ were on Medicaid, and $32 \%$ $(330,879)$ were under 18 years old. ${ }^{9}$ In addition, $63.4 \%$ of the patients lived at the poverty level and $36.5 \%$ lived below the poverty level. ${ }^{9}$

Securing off-site specialty referrals for $\mathrm{CHC}$ patients with chronic and acute conditions remains a key challenge ${ }^{3,7}$ and underscores the limited access to care for underserved populations. Limited access to health services predisposes vulnerable populations to fragmented care and lack of continuity of care. ${ }^{10,11}$ In addition, lack of timely access to needed SC often leads to complications, extended hospitalizations, and poor health outcomes, ${ }^{7}$ situations that can diminish the high-quality care that is characteristic of CHCs.

Previous studies have examined $\mathrm{CHC}$ patients and access to $\mathrm{SC}$ from different perspectives. For example, evaluations have been conducted from the points of view of executive directors and medical directors of CHCs., ${ }^{3}$ A study of Medicare enrollees was conducted to determine the association between care coordination and continuity of PC. ${ }^{13}$ Other studies have examined patients' perceptions of how their care was coordinated between PC providers (PCPs) and SC providers (SCPs). ${ }^{14} \mathrm{An}$ analysis was also done of physicians' satisfaction with care referral and coordination between pediatricians and specialists. ${ }^{15}$ While these scholarly inquiries have helped to create a broad understanding of health care access issues for vulnerable populations, studies on barriers to accessing $\mathrm{SC}$ for $\mathrm{CHC}$ patients from the perspectives of frontline personnel who take on the day-to-day responsibilities of coordinating SC referrals are not well documented and are relatively uncommon. This study thus fills this gap by examining the perspectives of $\mathrm{CHC}$ referral coordinators (RCs) who understand the nuts and bolts of the process.

\section{Materials and methods Sample and setting}

For this descriptive qualitative study, a convenience sample of 37 RCs representing 37 CHCs in Washington State was included. Six major counties in eastern, western, and central Washington were represented. Three clinics were in rural areas, far from major cities. The rest of the CHCs were located in urban and suburban areas. RCs are defined broadly in this study as personnel that oversee and coordinate $\mathrm{SC}$ referrals in CHCs. They include clinic managers, clinic directors, and licensed or unlicensed individuals hired as RCs. Participants' academic qualifications range from associate degree to doctorate.

The general inclusion criteria were that participants must be in charge of and oversee patient flow in the facility and/ or be actively involved in decisions regarding SC referrals and care coordination, be in the coordinating role in the facility for at least 3 months prior to the study, work at least half-time in the facility, and possess a degree in nursing or in other health-related fields such as social work or public health. Both men and women of diverse racial and ethnic backgrounds were represented in the participant pool based on the inclusion criteria. The basic demographic characteristics of the participants are presented in Table 1.

Table I Basic participant demographic characteristics $(n=37)$

\begin{tabular}{ll}
\hline & $\mathbf{n}$ \\
\hline Sex & 35 \\
Female & 2 \\
Male & \\
Education/highest education level & 2 \\
Doctorate & 5 \\
Master's & 22 \\
Baccalaureate & 8 \\
Associate & \\
Designated clinic roles & 23 \\
Clinic manager & 9 \\
Referral coordinator/patient-care coordinator & 5 \\
Clinic director & \\
Years in current role & \\
3 months-1 year & 5 \\
I-3 years & 15 \\
3-6 years & 9 \\
6-9 years & 5 \\
>10 years & \\
Clinic affiliations & 3 \\
Urban/suburban & \\
Rural & 34 \\
\hline
\end{tabular}




\section{Data collection}

Email and telephone requests for participation in the study were sent to potential participants. Clinic names and potential participants' contact information were obtained from the Washington State Department of Health. Among those who agreed to participate in the study, arrangements for face-toface or telephone interviews were made for data collection. Participants who were not able to meet for face-to-face interviews engaged in telephone interviews. Data were collected through semistructured face-to-face and phone interviews that lasted 45-60 minutes. Interviews included open-ended questions (Figure S1). Thirty interviews were audio-recorded. Seven participants declined audio-recording of their interviews; however, detailed notes were taken.

\section{Ethical consideration}

Approval for use of human participants for this study was obtained from the Institutional Review Board, Human Subjects Division, University of Washington. Prior to each interview, detailed information about the study and procedures was provided to all participants. All participants' questions were answered, and written informed consent was obtained from each participant. For participants who were interviewed by phone, informed consent was emailed to them, and scanned copies of signed consents were returned to the researcher.

\section{Data analysis}

Qualitative data from participant interviews were analyzed using content analysis guidelines. ${ }^{16,17}$ Interview transcripts and field notes were transcribed verbatim and reviewed by the author for accuracy. Units of analysis were extracted from the interview texts and field notes, and then put together into a single text. Categories of data related to the research questions were sorted and filtered from the units of analysis. Themes and subthemes pertaining to processes and barriers to $\mathrm{SC}$ access for $\mathrm{CHC}$ patients emerged from this reduction process and constitute the findings of the study.

\section{Results}

\section{Specialty-care referral/coordination process}

Each clinic highlighted in this study has standard protocols for SC referral. The participants described the details of the referral process, which starts with the $\mathrm{PCP}$ at the $\mathrm{CHC}$. When a PCP sees a patient and determines that consultation with an SCP is required, a referral order is written in a paper chart or in the electronic medical record (EMR) by the PCP.
This order goes to the RC. If the RC is not a licensed clinician, the nurse reviews and signs off on the order before the $\mathrm{RC}$ acts on the order. The $\mathrm{RC}$ evaluates the reason for the referral, type of specialist needed, type of insurance carried by the patient, and whether it is a new or established patient referral to a new or established SCP.

Methods of communication differ across clinics, and range from phone calls and faxes to electronic systems. One participant summed it up this way:

The referral process varies for different clinic networks, and could be quite as simple as picking up the phone and calling around, or it could be sophisticated and yet simplified through the use of the electronic medical record systems, such as EPIC, where the referral request from the provider drops into an electronic referral work queue.

The RC discusses the specialty referral with the patient to confirm interest in pursuing the referral. Once the patient acknowledges interest in the referral, an RC noted "We look for the right fit, considering where they live, who is accepting their insurance, zip-code parameters for some specialists when relevant, and continuity of care." A manager stated "If the patient is used to seeing a particular doctor, we try to send him back to the same one. Established care and continuity of care is very important to us, but sometimes it is not approved by insurance."

The RC identifies and contacts appropriate and available SCPs to confirm that the provider will see the patient. The $\mathrm{RC}$ also obtains preauthorization from the insurance if the patient is insured, then notifies the patient and sends the referral order and other required documents, such as laboratory and diagnostic results, to the specialty clinic for processing. Depending on the clinic, patient preference, and level of urgency, the RCs can make the appointment on behalf of the patient or the patient can call the clinic and set up his/her own appointment. For urgent referrals, however, participants noted that they process the referrals quickly (usually within 1-3 days), schedule the appointment, and then contact the patient. A third of the respondents reported that routine referrals take an average of 1 week from the time the referral was ordered to the time the appointment was scheduled. The average time from referral to actual specialist visit is 1-4 weeks. These timelines are variable however, often extending 6-12 months due to unique challenges associated with each referral. Furthermore, some referrals are often not tracked, since most PCPs do not always receive timely consultation reports from specialists and there are long waiting times for high-demand specialties with limited providers. The process 
of referral ends when the patient sees the SCP and the PCP receives a record of the specialty visit.

\section{Barriers to access to specialty care}

This study reveals several patient- and system-related barriers that limit access to SC for CHC clients. These are summarized in Table 2.

\section{Patient-related barriers}

\section{Socioeconomic factors}

A participant noted that although income does not determine access to $\mathrm{CHC}$ services because these clinics are for people of all income levels, it is the most potent factor that prevents people from getting SC. Participants identified poverty as a correlate of poor education, unemployment, underemployment, mental health issues, unmanaged chronic illnesses, and lack of health insurance, all of which impact people's ability to get SC. They also agreed that cost or inability to pay upfront at specialty clinics is a huge barrier that deters uninsured and underinsured patients from pursuing and completing their SC referrals. A participant explained it this way: "The economic factors create fear in patients and make them not schedule their appointments, because the sliding scale they get at the CHC is not applicable at the specialty clinic." Another participant reported that around 5\% of their clinic patients have decreased literacy and thus find it challenging to understand directions or the importance of going to the referred specialists. These highlight how different dimensions of socioeconomic factors impact SC for vulnerable CHC populations.

\section{Insurance}

Patient insurance was identified by $86 \%$ of participants as the most important driver of poor access to SC. Respondents reported that lack of insurance or not having the right insurance that is accepted at specialty practices is the biggest issue facing $\mathrm{CHC}$ patients. A respondent said that although their patients may encounter challenges related to cost, language, transport, and clinic location, care coordinators can

Table 2 Barriers to access specialty care

\begin{tabular}{ll}
\hline Patient-related & Socioeconomic factors \\
barriers & $\begin{array}{l}\text { Insurance } \\
\text { Transportation/clinic location } \\
\text { Language }\end{array}$ \\
Health system & $\begin{array}{l}\text { Shortage of personnel and lack of clinic-hospital } \\
\text { barriers }\end{array}$ \\
& $\begin{array}{l}\text { affiliation } \\
\text { Clinic-to-clinic communication gaps }\end{array}$ \\
& Limited availability of pro bono specialty providers \\
\hline
\end{tabular}

accommodate all of those factors, but not insurance. "The $\mathrm{CHC}$ is their insurance", noted another participant. Beyond the CHCs, the patients have limited options for specialized care. The findings also show that there are not enough specialists in certain areas of the state who are willing to accept CHC patients, and if one of them is accepting patients, they often have a long waiting list and take up to 6 months to see a patient. In addition, lengthy preauthorization processes from insurance companies delay care for patients.

\section{The uninsured}

According to RCs, securing SC referrals for uninsured patients is a huge challenge tied to financial barriers, since most patients do not have enough money to pay for the services. The patient's ability to pay a portion of the fee upfront for SC services is limited. A respondent explained this challenge:

So let's say they need to go see a urologist. The urology office is saying "You're uninsured? Fine, we'll see you, but you need to have $\$ 200$ down." The barrier comes with whether or not the patients can afford it.

Furthermore, the logistics of location play an important limiting role in referral and access to SC. Participants noted that there are some SCPs who are willing to accept uninsured patients only if they live within a certain mile radius of that specialty clinic or within certain zip codes. This further shrinks the pool of SCPs that is available to the uninsured. They also reported that it is difficult for patients to gain specialty access through charity care, due to the limited number of volunteer providers and subsequent long waiting times.

\section{Insured/underinsured and Medicare/Medicaid patients}

This study reveals that having certain insurance coverage often present even greater problems for the insured. Government programs (Medicare and Medicaid) and most managed-care plans do not translate into easier access to SC for patients. A participant put his views this way:

Our biggest challenge in the referral process is finding specialty care that will accept our patients, whether insured or uninsured. Mostly, the insured is where the largest barriers are, because a lot of the Medicare and Medicaid plans we accept are not contracted with the local specialists.

A manager stated:

The biggest challenge is that a good number of our patients have insurance that the specialists won't accept. We can refer them, but some specialists will not take them or they 
have to be referred to a practice that is over 100 miles away

. . . because local specialists are not available, unable, or unwilling to accept the patients.

Care coordinators in rural areas noted that their patients are heavily impacted by this issue, as they often struggle to get to specialists in major cities who are willing to accept their insurance plans. Those in the urban areas also reported that $\mathrm{CHC}$ in cities with a high number of Medicaid/Medicare homeless populations also encounter difficulties linking them with SC. On the contrary, respondents noted that SC coordination and referral for $\mathrm{CHC}$ patients who hold preferred health insurance plans through their jobs are very easy. However, some patients often do not have enough money to pay for out-of-pocket costs, and in some cases do not keep their appointments.

\section{Transport/clinic location}

Transport is another impactive barrier to access to SC, according to $68 \%$ of respondents. A respondent summarized it this way: "Transportation is a big issue, due to our patients' limited resources. If we can get patients covered to see a specialist and yet they cannot get there, we have not solved the problem of access." Participants noted that most of their patients do not drive. For patients in rural areas, including those that live in islands with limited specialty services, it is extremely difficult to travel long distances. A respondent noted that patients often miss appointments because the bus or transport service could not bring them on time and they could wait for hours after their appointments to get home. These factors result in frustration and lack of follow-through with hard-fought medically necessary appointments.

\section{Language}

Language impacts communication and quality of care related to SC. Participants in clinics located in areas with high populations of new immigrants, including refugee-relocation areas, reported language to be an important barrier to access. They noted that patients are not refused access to care due to their inability to speak English. However, communication, understanding, and compliance are significantly impacted. For example, language is a huge factor in missed appointments, due to scheduling miscommunications and not understanding referral instructions, which could influence patient compliance.

Despite the fact that language constitutes a challenge in the coordination and referral process, all participants agreed that it is not a deal breaker. A respondent stated: "Although language is an issue in patient care, no one is denied care based on language. It is against the law; it is discrimination." The majority of participants reported that their clinics effectively manage language barriers by using interpreters and hiring bilingual and multilingual employees. Respondents from clinics that serve a large number of the Spanish population reported language as a nonfactor, since the majority of their employees speak Spanish.

\section{Health system barriers \\ Shortage of personnel}

Findings show that the majority of SC services are provided outside the CHCs, and access to those services are challenging for patients. All participants agreed that there are not enough SCPs that are available to CHC patients. A manager put it this way:

There is an extreme lack of specialists available to us. It's very, very hard to find specialists, especially those who need to see a patient in a timely manner. Many times, our patients can't get in for 6-8 months.

A coordinator explained that a shortage of SCPs correlates with insurance and specific specialties. For example, a particular insurance may have only one or two providers in a particular specialty in certain areas, whereas others may have seven or eight providers. Examples of specialty services that are in high demand and often take longer periods of time and more effort to arrange include cardiology, orthopedics, neurology, gastroenterology, rheumatology, urology, gynecology, oncology, dermatology, ophthalmology, and chronic pain management. One participant said:

Neurologists, rheumatologists, and urologists? There are not many of them. There are either so many patients on their waitlist, maxed-out capacity, or they are not taking patients' insurance, or they do not offer charity care.

Another participant described the challenge of not having enough specialists as the "daily roadblocks to care", as many SCPs are often booked out in advance for several months.

Furthermore, a shortage of RCs at the CHCs was identified as a huge challenge, resulting in backlogs of unprocessed referrals. A participant reported that not having an adequate number of coordinators is a huge challenge. A manager put it this way: "We only have one referral coordinator, and most of her time is spent on the phone. We don't have the capacity to serve the need." Some RCs in high-traffic clinics, particularly in urban areas, reported being overwhelmed by high referral requests due to high patient volume. 
Clinic-to-clinic communication gaps and lack of clinichospital affiliations

Gaps and delays in communication between $\mathrm{CHCs}$ and $\mathrm{SC}$ clinics were identified by respondents as huge barriers to $\mathrm{SC}$ access and utilization. The communication gap is significant during the initial search for specialists and at the completion of consultation. Sixteen clinics represented in this study belong to larger CHC networks that use EMRs. These include NextGen, Epic, and AllScripts. Study participants who use these systems reported that the software systems' online referral forms facilitate and ease the patient-care coordination and referral process within their network. This means that coordinators can share information about the available pool of specialists in terms of who is accepting patients and who is not. However, outside their networks, different EMR systems do not communicate with each other. A respondent said, "Our electronic medical record systems don't talk to each other, so that's why we're all faxing." Participants expressed concern that RCs in these clinics spend huge amounts of time on the phone and fax to identify specialists who could see their patients.

The coordination and referral process is more challenging if the PCPs at CHCs have no affiliations with hospitals or specialty clinics. Only two clinics in the study were affiliated with hospitals. Participants reported that clinic-hospital affiliations ease the process for the RCs and patients, and help address the issue of finding a willing and available specialist, as well as minimize delays in SC access for patients. Moreover, communication between PCPs and specialists is easier within affiliated facilities. Participants expressed frustration with the lack of a follow-up system to track referral completions with providers in unaffiliated clinics. One participant said:

The specialists are not very good at sending us the notes back, even though we request on our referral forms to send the results of the visit to us within a week. Sometimes, the specialists require the patient to sign a release form to allow specialists to release the records to us, even though we are the ones that referred them to the specialist in the first place. Everyone sort of has a different interpretation of what HIPPA [Health Insurance Portability and Accountability Act] is.

Another participant stated:

When the specialty appointment happens, there are not often follow-ups afterwards. I think that the ball drops there. If we could get the specialist's notes back with the medical changes made, and plan of care, etcetera, that would be better for our patients.
The majority of participants admitted that they did not usually track the number of their patients who were referred to SC that actually got such care. Some of their reasons for this were lack of time and lack of communication from the specialists to the referring PCP regarding the outcome of the specialty visit.

\section{Limited availability of pro bono specialty providers}

Specialist volunteers help fill some specialty access gaps for patients who would otherwise not afford such care by donating their time and resources. Some RCs reported that their patients benefit from individual volunteer specialists who occasionally visit their clinics to provide specialty services in behavioral health, dental care, podiatry, and women's health. One respondent noted that a volunteer urologist visits her clinic once a month, and another reported that a volunteer optometrist visits her clinic occasionally to conduct diabetic retinal screenings for their patients.

Participants from three counties hailed Project Access Northwest (PANW) as their big resource for securing SC for their clients. One participant put it this way:

This program called Project Access Northwest ... is a gateway for patients to get in to see specialists. They set up a database of providers in specialty offices ... and ask providers if they will volunteer their time to see patients on a charity care basis.

PANW is a nonprofit organization that partners with hospital systems and multispecialty medical groups throughout King, Kitsap, and Snohomish counties to provide SC to the uninsured and underinsured in Washington State. The organization matches volunteer specialists with carefully prescreened patients in need of care. ${ }^{18}$ Participants noted that although PANW is a very reliable resource for their patients, it is only one organization and is for nonurgent cases, as they have a lengthy average waiting time, between 6 months and 1 year.

\section{Discussion}

For vulnerable low-income clients who utilize CHCs as their safety net, access to $\mathrm{SC}$ remains a huge challenge. There is clear disparity in SC access for the uninsured and underinsured. This study shows that for most $\mathrm{CHC}$ patients requiring $\mathrm{SC}$, coordinating their referrals is beset by multifaceted barriers. Care coordination is multidisciplinary and is central to quality health care access. It involves deliberate organizing and sharing of information among all individuals concerned with a patient's care. ${ }^{19}$ Coordinating patient care involves effective relay of information across settings, with 
less repetition of information and tests and more timely and effective delivery of needed services. ${ }^{20}$ In the case of Washington State, this study shows that information sharing and referrals are not timely.

Although anyone can see a specialist, not everyone can pay for the services. Income is the salient undercurrent that sets the stage for all the other patient factors that impact access and utilization of SC services. Getting to specialists in the first place is often challenging for most $\mathrm{CHC}$ patients, due to transport or location issues, in addition to their inability to pay for the services. Even when SCPs are in adequate supply and are available and willing to see low-income patients, access may still not be achieved. Access is measured in terms of utilization and is dependent on affordability, physical accessibility, and acceptability of the services, and not merely adequacy of supply. ${ }^{21}$

Poverty or low socioeconomic resources intensify disparities in access to $\mathrm{SC}$, which is often more expensive than PC. This study clearly shows that access to $\mathrm{CHCs}$ is not income-dependent. However, there is a correlation between poverty and financial hardships associated with paying for medical care outside CHCs. Data from the National Center for Health Statistics show that families with lower incomes are more likely to experience financial burdens of medical care. ${ }^{22}$ For example, families with incomes at or below $250 \%$ of the federal poverty level have the highest levels of any financial burden of medical care. Also, families that have children or an uninsured family member stand the risk of such financial burden. ${ }^{22}$ These financial challenges of paying medical expenses are generally cumulative and often push families deeper into poverty and poorer health.

Scholarly review of the link between poverty and mental health suggests that much of the risk for mental health problems can be linked to higher levels of stress among individuals and families living in poverty. ${ }^{23,24}$ These individuals and families face numerous logistical, attitudinal, and systemic barriers to obtaining mental health services, such as transportation, childcare, lack of health insurance, and difficult work hours. ${ }^{23}$ Other stressors they face in addition to economic strain include discrimination and violence, frequent moves and transitions, and other traumatic experiences, ${ }^{23}$ as well as food insecurity and housing problems. ${ }^{24}$

A significant relationship also exists between poverty and unmanaged chronic diseases. A previous study showed that poverty negatively impacts the health care of populations with chronic disease conditions. Based on a multidimensional framework, poverty is not based only on income but also captures deprivation in health, livelihood (education and employment), standard of living, and housing. ${ }^{25}$ For example, for older adult patients with chronic kidney disease who also live in poverty, research shows that they experience barriers to treatment and renal diet adherence, limited transportation, lack of affordable housing, and reliance on public medical insurance. $^{26}$

A key socioeconomic barrier to SC access and utilization that is central to the findings of this study is decreased health-literacy skills of some CHC patients. Health literacy is multidimensional and is the degree to which individuals have the capacity to obtain, process, and understand basic health information and services needed to make appropriate health decisions. ${ }^{27}$ Evidence shows a consistent association between low health literacy and limited health-related knowledge and comprehension, which impacts the use of health care services, and disparities in health outcomes. ${ }^{28}$ Health literacy is also the capacity of an individual to understand information related to a disease, in order to make an informed decision. ${ }^{29}$ For example, health literacy is critical for patients with chronic conditions, such as kidney disease, in order to understand the complexity of the disease and self-management skills, such as adhering to dietary restrictions and complex medication regimens. ${ }^{28,29}$ For chronically ill CHC patients with limited knowledge of their disease conditions, their health outcomes might be negatively impacted.

Health-insurance literacy could affect SC access. Knowledge of the health system and complexities of health-insurance coverage can be challenging. For example, in the era of the Patient Protection and Affordable Care Act (ACA), although the mandate requires all residents with legal documents to have health insurance, there is no mechanism or provision for educating potential enrollees or the public about healthinsurance options or an appropriate health insurance plan. ${ }^{30}$ For example, navigating the health-insurance marketplace to identify coverage options for SC and out-of-pocket costs can pose challenges for patients. Improved access to health information, services, and care are foundational to SC access.

A key finding of this study is that government programs, such as Medicaid, carried by most CHC patients in Washington State, are not popular among specialists in terms of acceptability. This is supported by a previous study that indicated that cuts in Medicaid physician fees led to statistically significant reductions in the number of visits for Medicaid patients compared to privately insured patients. ${ }^{31}$ Another study showed that Medicaid patients had the longest average waiting times for SC than any other insurance group, while patients with private insurance had the shortest average waiting time. ${ }^{32}$ Data from the US Department of 
Health and Human Services show that in 2009-2010, generalist physicians had shorter waiting times for appointments compared with SCPs. ${ }^{33}$ RCs spend a significant amount of time searching for and matching SCPs with patients who are often wait-listed. Delays in access to specialty consultation and treatment can have negative effects on patients' health outcomes.

The use of telephone consultations (teleconsultations) or electronic consultations (e-consultations) could provide faster access to specialist expertise without going through the complexity of setting up clinic appointments. Evidence shows that telehealth reduces consultation delays between PCPs and specialist CPs and improves access to specialized care. ${ }^{34}$ Also, e-consultations as an alternative to in-person SC referral is useful in interpreting results, defining next steps for diagnosis and additional tests, and referring patients for procedures..$^{35}$ Evidence shows that e-consultations are easy to use and increase the timeliness of access to SC, due to the rapidity of specialist response and the provision of a mechanism for asking clinical questions. ${ }^{36}$ Despite legal concerns, fewer face-to-face consultations with specialists, and workflow and workload issues, PCPs believe that e-consultations result in more efficient medication management, expedited diagnostic testing, and more efficient communication with specialists. ${ }^{36}$

Having common EMRs facilitates e-consultations between providers. Common EMRs were not utilized by many CHCs highlighted in this study. Poor communication and coordination are amplified by EMRs that do not talk to other systems. Previous research concurs with this finding that uneven adoption of nonstandardized, noninteroperable EMR systems only delay the chance to move more closely to a transformed health care system. ${ }^{37}$ Further evidence shows that some EMRs are less able to support coordination between clinicians and settings, in part due to their design and a lack of standardization of key data elements required for information exchange. ${ }^{38}$ For higher-risk patients, case management systems help coordinate workflow, including communication between multiple specialists and patients. ${ }^{37}$ Improved communication and care coordination translates into reduced cost of health care services and improved health outcomes for patients.

This study shows that coordination and access to SC is enhanced when PCP and SCP practices are connected. Clinic-hospital affiliations are beneficial to patients and $\mathrm{RCs}$, as they speed up the exchange of information. CHCs that have affiliations with hospitals provide their patients with easier access to specialized services in those hospitals.
Evidence shows that the primary objectives of health system affiliations are to integrate care in the community, eliminate duplicative services, reduce costs, and standardize work processes, while encouraging service excellence. ${ }^{39}$ In an environment where many specialists are unwilling to see underinsured and uninsured patients, hospital affiliations facilitate the process and often guarantee successful specialty referral and appointment. Health care communication that is eased by effective use of EMRs and clinic affiliations will significantly reduce delays in SC access and improve health outcomes for vulnerable populations.

Although the goal of the ACA is to address access to health care by reducing the cost of care and making it affordable, there is however no provision under the law that states that a physician and other providers must accept any patient's form of coverage.$^{40}$ As a result, increased coverage through Medicare and Medicaid expansions, which Washington State residents have benefited from, has led to increased numbers of patients seen at safety-net clinics, including CHCs. However, there has been no corresponding increase in the number of patients accepted for care by specialists. Clearly, there is nothing like low-cost SC. The need for SC is still not met for underserved populations.

Specialist availability is a critical determinant of access. This study emphasizes the limitations to access faced by patients, due to the inadequate number of available specialists. The Association of American Medical Colleges reported a significant shortage among many non-PC specialties from 2015 to $2030 .^{41}$ They projected that by 2030 , non-PC specialties will experience a shortfall of between 33,500 and 61,800 physicians in the US..$^{41}$ The association also projected that if barriers to utilization were removed for underserved patients, the US would need up to 96,000 doctors to meet patient needs between 2014 and $2025 .{ }^{42}$

Alongside specialist shortages is the inadequate number of RCs. As a result, many CHCs experience backlogs of patient referrals. RCs do the bulk of the work and are the heavy lifters in the referral and coordination process. Their activities stretch from the time the PCP writes the initial referral order to the time the referral is completed. They are key personnel in the coordination and referral process and act as multiway contacts for patients, family members, the PCP, specialists, insurance companies, and other ancillaryservice providers. In short, RCs are the glue that connect all the pieces of the referral system together. The effectiveness and efficiency of care coordination hinge on the RCs. A report showed that for CHCs that employed RCs, referral completion and written communication from specialists to referring 
clinicians were present in medical records of nearly $75 \%$ of referral cases. ${ }^{32} \mathrm{RCs}$ help to promote effective care delivery by facilitating communication among the CHCs, specialty clinics, and the patients. A shortage of RCs results in communication breakdown, poor coordination of care between providers, and poor patient outcomes.

\section{Recommendations}

Barriers to SC access for CHC patients outlined in the findings of this study are interlocking. Some recommendations for improvement were articulated throughout the "Discussion" section and include:

- effective coordination of patient care among all service providers throughout the care continuum ${ }^{20,43}$

- CHC-hospital affiliations to facilitate care coordination and referral and to reduce fragmentation of care delivery $^{39}$

- availability of standardized, interoperable EMR systems, including the use of e-consultations and teleconsultations to mitigate delays in $\mathrm{SC}$ access ${ }^{34-37}$

- affordable comprehensive health-insurance coverage that guarantees access to both PC and SC for low-income CHC patients $^{44}$

- increasing the number of specialists and $\mathrm{RCs}^{32,42}$

- improving health literacy related to knowledge of disease management, health insurance coverage options, and general health system functioning ${ }^{29,30}$

- tackling the core challenges of poverty and its associated links to poor education, unemployment, lack of appropriate insurance coverage, transportation, low health literacy, poor chronic conditions, and mental health issues.

\section{Study limitations and next steps}

The study has limited geographic variability. Care coordinators in urban clinics in Washington State constitute the majority of participants. Because the study focused on the RCs' perceptions of barriers to SC coordination and the referral process, the researcher did not collect demographic information from the clinics that RCs were affiliated with. The researcher also recognizes the significance of presenting a breakdown of the proportion of patients in each insurance category for each clinic. This breakdown was challenging to calculate and assign values for, because the information was provided in a generalized way. Specific numbers of patients served at each clinic and the number of patients with conditions that required SC referral were solicited, but the captured numbers were broad and ranged from daily and weekly to monthly. The collected data were difficult to interpret in a meaningful way and were thus not included in the findings. Furthermore, CHC patients were not included in this study, because the research was designed to reflect the views of RCs who help facilitate SC referrals for patients.

This study provides new evidence regarding the challenges facing $\mathrm{CHC}$ patients in accessing SC services. It also provides baseline data for future research on a more expansive interventional study on $\mathrm{SC}$ access for vulnerable populations. Further follow-up study recommendations include exploration of the perspectives of $\mathrm{CHC}$ patients on the difficulties they encounter in accessing the services of specialists; exploration of the views of specialists and PCPs on the challenges they encounter in supporting $\mathrm{CHC}$ patients who require SC services, and their recommendations for improvement; and examination of how SC coordination and referral for $\mathrm{CHC}$ patients have been impacted following the implementation of the ACA.

\section{Conclusion}

This study describes the processes and barriers to SC access for vulnerable populations in CHCs. Understanding the complexities and challenges surrounding $\mathrm{SC}$ coordination and access helps to determine the health system's needs in providing comprehensive and uninterrupted access to quality health care for all citizens. This study clearly shows that increased access to $\mathrm{PC}$ at $\mathrm{CHCs}$ has not translated into improved access to SC. Failure to fill this gaping hole in our health care system will result in persistent inequity in access to comprehensive health care services. There is a need for effective policy strategies that enhance care coordination with all partners along the health care delivery continuum. An integrative model of care that provides seamless connection between PCPs and SCPs must be established to ensure continuity of care for all and to decrease the overall burden of disease, morbidity, and mortality.

\section{Acknowledgments}

The author thanks the community health clinic personnel who participated in this study. Thanks also goes to Matt Todd, Ladna Fara, and Henry Rawner for their help in data collection. In addition, gratitude is extended to Chigozie Allison Ezeonwu for her help in editing this manuscript.

\section{Disclosure}

The author reports no conflicts of interest in this work. 


\section{References}

1. Schauffler HH, Wolin J. Community health clinics under managed competition: navigating uncharted waters. J Health Polit Policy Law. 1996;21(3):461-488.

2. Ferrer RL. Pursuing equity: contact with primary care and specialist clinicians by demographics, insurance, and health status. Ann Fam Med. 2007;5(6):492-502.

3. Cook NL, Hicks LS, O’Malley AJ, Keegan T, Guadagnoli E, Landon BE. Access to specialty care and medical services in community health centers. Health Aff (Millwood). 2007;26(5):1459-1468.

4. Gusmano MK, Fairbrother G, Park H. Exploring the limits of the safety net: community health centers and care for the uninsured. Health Aff (Millwood). 2002;21(6):1888-1894.

5. Weissman JS, Moy E, Campbell EG, et al. Limits to the safety net: teaching hospital faculty report on their patients' access to care. Health Aff (Millwood). 2003;22(6):156-166.

6. Cook NL, Ayanian JZ, Orav EJ, Hicks LS. Differences in specialist consultations for cardiovascular disease by race, ethnicity, gender, insurance status, and site of primary care. Circulation. 2009;119:2463-2470.

7. Adashi EY, Geiger HJ, Fine MD. Health care reform and primary care: the growing importance of the community health center. $N$ Engl J Med. 2010;363(22):2047-2050.

8. Washington Association of Community and Migrant Health Centers. Washington State community health centers. 2015. Available from: http://nativeproject.org/upload/userfiles/CommunityPartners/2014_ WA_State_Fact_Sheet_-_Final.pdf. Accessed September 17, 2017.

9. Bureau of Primary Health Care. 2016 Health center data: Washington data. 2016. Available from: https://bphc.hrsa.gov/uds/datacenter. aspx?q=tall\&year=2016\&state=WA. Accessed September 9, 2017.

10. Naylor M, Keating SA. Transitional care. Am J Nurs. 2008;108(9 Suppl):58-63.

11. Coleman EA, Min S, Chomiak A, Kramer AM. Posthospital care transitions: patterns, complications, and risk identification. Health Serv Res. 2004;39(5):1449-1465.

12. Neuhausen K, Grumbach K, Bazemore A, Phillips R. Integrating community health centers into organized delivery systems can improve access to subspecialty care. Health Aff (Millwood). 2011;31(8): $1708-1716$.

13. Liss DT, Chubak J, Anderson ML, Saunders KW, Tuzzio L, Reid RJ. Patient-reported care coordination: associations with primary care continuity and specialty care use. Ann Fam Med. 2011;9(4):323-329.

14. Cunningham PJ. Beyond parity: primary care physicians' perspectives on access to mental health care. Health Aff (Millwood). 2009;28(3):w490-w501.

15. Forrest CB, Glade GB, Baker AE, Bocian A, Von Schrader S, Starfield B. Coordination of specialty referrals and physician satisfaction with referral care. Arch Pediatric Adolesc Med. 2000;154(5):499-506.

16. Graneheim UH, Lundman B. Qualitative content analysis in nursing research: concepts, procedures and measures to achieve trustworthiness. Nurse Educ Today. 2004;24(2):105-112.

17. Elo $\mathrm{S}$, Kyngas $\mathrm{H}$. The qualitative content analysis process. $J A d v$ Nurs. 2008;62(1):107-115.

18. Project Access Northwest. About us. 2017. Available from: https:// projectaccessnw.org/overview/about-us. Accessed September 11, 2017.

19. Agency for Healthcare Research and Quality. Care coordination. 2014. Available from: https://www.ahrq.gov/professionals/prevention-chroniccare/improve/coordination/index.html. Accessed September 11, 2017.

20. Lamb G. Care coordination, quality, and nursing. In: Lamb G, editor. Care Coordination - The Game Changer: How Nursing is Revolutionizing Quality Care. Silver Spring (MD): Nursesbooks; 2013:3-4.

21. Gulliford M, Figueroa-Munoz J, Morgan M, et al. What does 'access to health care' mean? J Health Serv Res Policy. 2002;7(3):186-188.

22. Cohen RA, Kirzinger WK. Financial burden of medical care: a family perspective. NCHS Data Brief. 2014;(142):1-8.
23. Santiago CD, Kaltman S, Miranda J. Poverty and mental health: how do low-income adults and children fare in psychotherapy? J Clin Psychol. 2013;69(2):115-126

24. Hodgkinson S, Godoy L, Beers LS, Lewin A. Improving mental health access for low-income children and families in primary care setting. Pediatrics. 2017;139(1):e20151175.

25. Dhongde S, Haveman R. Multi-dimensional poverty in the US. 2014. Available from: https://www.irp.wisc.edu/newsevents/seminars/ presentations/2013-2014/US_MPI_dhongde_haveman.pdf. Accessed November 6, 2017.

26. Caplan MA, Washington TR, Swanner L. Beyond income: a social justice approach to assessing poverty among older adults with chronic kidney disease. J Gerontol Soc Work. 2017;60(6-7):553-568.

27. Nielsen-Bohlman L, Panzer A, Hamlin BN, et al. Health literacy: a prescription to end confusion. 2004. Available from: https://www.nap. edu/read/10883/chapter/1\#vii. Accessed November 7, 2017.

28. Berkman ND, Sheridan SL, Donahue KE, Halpern DJ, Crotty K. Low health literacy and health outcomes: an updated systematic review. Ann Intern Med. 2011;155(2):97-107.

29. Jain D, Green JA. Health literacy in kidney disease: review of the literature and implications for clinical practice. World J Nephrol. 2016;5(2):147-151.

30. Brown V. Infusing adult education principles into a health insurance literacy program. Health Promot Pract. Epub 2017 Apr 1.

31. Decker SL. Changes in Medicaid physician fees and patterns of ambulatory care. Inquiry. 2009;46(3):291-304.

32. Patrick G, Bisgaier J, Hasham I, Navarra T, Hickner J. Specialty care referral patterns for the underserved: a study of community health centers on the south side of Chicago.J Health Care Poor Underserved. 2011;22(4):1302-14.

33. Hing E, Schappert SM. Generalist and specialty physicians: supply and access, 2009-2010. NCHS Data Brief. 2012;(105):1-8.

34. Marcolino MS, dos Santos JP, Neves DS, Alkmim MB. Teleconsultations to provide support for primary care practitioners and improve quality of care: experience of a large-scale telehealth service in Brazil. Stud Health Technol Inform. 2015;216:987.

35. Fort MP, Namba LM, Dutcher S, et al. Implementation and evaluation of the safety net specialty care program in the Denver metropolitan area. Perm J. 2017;21:73-81.

36. Gupte G, Vimalananda V, Simon SR, Devito K, Clark J, Orlander JD. Disruptive innovation: implementation of electronic consultations in a Veterans Affairs health care system. JMIR Med Inform. 2016;4(1):e6.

37. Hillestad R, Bigelow J, Bower A. Can electronic medical record systems transform health care? Potential health benefits, savings, and costs. Health Aff (Millwood). 2005;24(5):1103-1117.

38. O'Malley AS, Grossman JM, Cohen GR, Kemper NM, Pham HH. Are electronic medical records helpful for care coordination? Experiences of physician practices. $J$ Gen Intern Med. 2009;25(3):177-185.

39. Schneider M. Affiliation and its benefits to the hospital and community. Nurs Admin Q. 2016;40(4):342-345.

40. Bindman AB, Coffman JM. Calling all doctors: what type of insurance do you accept? JAMA. 2014;174(6):869-870.

41. Association of American Medical Colleges. The complexities of physician supply and demand: projections from 2015 to 2030. 2017. Available from: https://aamc-black.global.ssl.fastly.net/production/ media/filer_public/a5/c3/a5c3d565-14ec-48fb-974b-99fafaeecb00/ aamc_projections_update_2017.pdf. Accessed September 18, 2017.

42. Association of American Medical Colleges. The complexities of physician supply and demand: projections from 2014 to 2015. 2016. Available from: https://www.aamc.org/download/458082/data/2016_complexities_ of_supply_and_demand_projections.pdf. Accessed September 18, 2017.

43. Bodenheimer T. Coordinating care: a perilous journey through the health care system. $N$ Engl J Med. 2008;358(10):1064-1071.

44. Miller S, Wherry LR. Health access to care during the first 2 years of the ACA Medicaid expansions. N. Engl J Med. 2017;376(10):947-956. 


\section{Supplementary material}

I. What best describes your job title? What is your role in the referral process? How long have you been in your current position?

2. What is your educational level?

3. How would you describe your clinic location, urban or rural?

4. On average, how many patients are seen in your clinic daily? Weekly? Monthly? In the last month? In the last 3 months?

5. Thinking of all the patients seen at your clinic in the last month, approximately what percentage of your clients have the following insurance categories? Medicaid? Medicare? Private insurance? No insurance?

6. What proportion of patients seen at your $\mathrm{CHC}$ have chronic conditions?

7. What proportion of patients with chronic conditions require referral for specialty care?

8. When a primary-care provider (doctor, registered nurse practitioner, physician assistant) requests specialty-care referral for a patient in your clinic, what do you do to initiate and coordinate the referral? What are the steps in your referral process?

9. Does your clinic have specific referral protocols or guidelines for specialty-care referral? If yes, what are the protocols?

10. What major challenges or roadblocks do you face in referring patients to specialty care? That is, from the time the provider orders a specialty-care consultation till the time the service is delivered, what challenges do you encounter?

11. What specific patient and clinic factors determine where you send the patients to receive specialty care?

12. What specialty-care services, if any, are available on-site at your clinic?

13. What specialties present more challenges than others in terms of getting timely referrals for patients?

14. Is your CHC part of a referral network, or have affiliations with any hospitals or medical centers? Please describe if yes.

15. On average, how long does it take to complete a referral?

16. How does your clinic track and receive reports from the specialists regarding patients' status and treatment? How do your clinic and the specialty clinics communicate with each other?

Figure SI Interview questions.

Abbreviation: $\mathrm{CHC}$, community health clinic/center.

Journal of Multidisciplinary Healthcare

\section{Publish your work in this journal}

The Journal of Multidisciplinary Healthcare is an international, peerreviewed open-access journal that aims to represent and publish research in healthcare areas delivered by practitioners of different disciplines. This includes studies and reviews conducted by multidisciplinary teams as well as research which evaluates the results or conduct of such teams or health 